\title{
Design considerations for an integrated microphysiological muscle tissue for drug and tissue toxicity testing
}

\author{
George A Truskey*1, Hardean E Achneck², Nenad Bursac', Hon Fai Chan', Cindy S Cheng', Cristina Fernandez', \\ Sungmin Hong ${ }^{4}$, Youngmee Jung' ${ }^{1}$, Tim Koves ${ }^{5}$, William E Kraus ${ }^{5}$, Kam Leong', Lauran Madden', William M Reichert ${ }^{1}$ \\ and Xuanhe Zhao ${ }^{4}$
}

\begin{abstract}
Microphysiological systems provide a tool to simulate normal and pathological function of organs for prolonged periods. These systems must incorporate the key functions of the individual organs and enable interactions among the corresponding microphysiological units. The relative size of different microphysiological organs and their flow rates are scaled in proportion to in vivo values. We have developed a microphysiological three-dimensional engineered human skeletal muscle system connected to a circulatory system that consists of a tissueengineered blood vessel as part of a high-pressure arterial system. The engineered human skeletal muscle tissue reproduces key mechanical behaviors of skeletal muscle in vivo. Pulsatile flow is produced using a novel computer-controlled magnetically activated ferrogel. The system is versatile and the muscle unit can be integrated with other organ systems. Periodic monitoring of biomechanical function provides a noninvasive assessment of the health of the tissue and a way to measure the response to drugs and toxins.

Keywords: microphysiological system, skeletal muscle myoblasts, tissue-engineered blood vessel, contractile force, oxygen gradients, differentiation
\end{abstract}

\section{Introduction}

In vitro microphysiological multiorgan systems using human cells provide a novel method to identify promising drug candidates [1]. Skeletal muscle is an important component of such systems because skeletal muscle

*Correspondence: gtruskey@duke.edu

'Department of Biomedical Engineering, Duke University, 136 Hudson Hall,

CB 90281, Durham, NC 27708-0281, USA

Full list of author information is available at the end of the article accounts for $40 \%$ of the body's mass and about $30 \%$ of the resting metabolic rate [2]. Under resting conditions about $20 \%$ of cardiac output passes through muscle, and during heavy exercise muscle requires as much as $80 \%$ of cardiac output. Muscle is responsible for about $75 \%$ of wholebody insulin-stimulated glucose uptake.

Sarcopenia, the age-associated loss of skeletal muscle mass, is a major consequence of aging and leads to lower metabolic adaptation, slowed immunological responses to disease, weakness, poor function and disability with advancing age [3]. The onset and progression of sarcopenia is a strong predictor of mortality and prevalence increases with advancing age [4]. Type 2 diabetes or lowgrade inflammation is more common in individuals who have sarcopenia [5]. Other major diseases that involve muscle include muscular dystrophy and neurodegenerative diseases.

Skeletal muscle is both a potential target for drug therapy and a site of drug toxicity. Treatments for type 2 diabetes affect and even target skeletal muscle metabolism as therapeutic mechanisms. Several candidate drugs are under investigation to treat sarcopenia, although special considerations are needed to define efficacy [6] and to ensure that side effects are limited [7].

Mitochondrial toxicity is a major reason why a drug may fail in clinical trials or is recalled after approval [8]. Like the liver and heart, skeletal muscle is affected by mitochondrial drug toxicity. Certain statins, used to treat dyslipidemia, can cause severe myopathy. Drugs known to cause mitochondrial toxicity, such as cerivastatin [9] and thiazolidinediones [8] (used to treat type 2 diabetes), also affect muscle function.

\section{Engineered three-dimensional human skeletal muscle tissue}

To evaluate functional changes to human skeletal muscle after exposure to drugs, we developed a microphysiological system consisting of highly aligned, functional, and metabolically active engineered human muscle and 
human tissue-engineered blood vessel (TEBV) (Figure 1). Myoblasts were obtained from biopsies of the vastus lateralis of healthy middle-aged volunteers, endothelial cells were cultured from blood-derived late outgrowth endothelial progenitor cells in human umbilical cord blood [10], and vessel wall medial cells were either human dermal fibroblasts or mesenchymal stem cells. These individual units are cultured separately for about 2 weeks to enable the skeletal myoblasts to fuse and differentiate and to allow the blood vessels to develop sufficient mechanical strength. The two engineered tissues are integrated into a perfusion system that enables monitoring of function.

Major challenges for in vitro culture of human skeletal muscle are the lack of efficient methods to differentiate large numbers of human muscle cells from induced pluripotent stem cells and the slower doubling time and rate of fusion for primary human skeletal muscle cells compared with mouse or rat myoblasts. Further, rat and mouse myotubes undergo spontaneous contractions in two-dimensional and three-dimensional cultures, whereas contractile human muscle fibers in vitro have only been obtained following co-culture with rat or human motoneurons [11].

We utilized our prior findings with engineered rodent muscles [12-18] to fabricate three-dimensional primary human skeletal muscle tissue bundles. Optimization of the dimensions of the muscle bundles, the media and hydrogel composition, the cell density, and the differentiation procedure (Figure 1) resulted in three-dimensional human muscle bundles with highly aligned, cross-striated myofibers containing myogenin-positive nuclei and acetylcholine receptor clusters (Figure 2b1). Functional properties of such engineered muscle were tested using standard force test protocols [17]. In response to single electrical stimuli, the bundles produced twitch contractions; at higher stimulation frequencies, the twitch responses fused into a more forceful tetanic contraction. Amplitudes of twitch and tetanus-specific force were significantly lower than those of native muscle, probably due to a lower muscle fiber density and a smaller fiber diameter [19], as well as the presence of immature forms of muscle proteins. Similar to native muscle, engineered human muscle bundles exhibited a positive Starling-like force-length relationship. After the necessary optimization, the method for engineering functional human muscle tissues is robust and reproducible and tissue bundles remain functional for 4 weeks.

To promote differentiation and the production of adult forms of muscle contractile proteins, and thereby increase contractile force [20], we have transfected myoblasts with miRNAs prior to formation of the engineered human muscle bundles. miR-133a and miR-696 are of particular interest because they affect important developmental processes: miR-133 a blocks myoblast proliferation by inhibiting serum response factor [20], and miR-696 inhibits mitochondrial biogenesis and oxidative metabolism by blocking the metabolic transcriptional co-activator PGC-1 $\alpha$ [21]. Engineered human skeletal muscle myoblasts were transfected with anti-miR-133a, with anti-miR-696, or with both antimiRNAs. The miRNA transfection produced longer and more aligned and cross-striated myofibers when compared with myoblasts that received a scrambled RNA sequence. Myoblasts transfected with both anti-miR133a and anti-miR-696 exhibited considerable slow myosin heavy chain, indicative of type I muscle fibers, and generated higher specific contractile forces compared with bundles prepared from myoblasts transfected with only a single anti-miRNA.

To further enhance differentiation, normal contractile function of skeletal muscle under various physical demands can be simulated by applying cyclic stretch or electrical stimulation (Figure 1, inset). Electrical stimulation is applied via two platinum electrodes and can be synchronized with the mechanical stimulation [14]. The electrical stimulation waveform is precisely bipolar and the amplitude of electrical stimulation is adjusted to accommodate potential changes in the excitation threshold expected to occur with the muscle maturation.

\section{Designing a microperfusion system for engineered skeletal muscle}

Engineered human muscle systems are used either as a dynamically conditioned standalone culture system to enable muscle maturation or as an integrated module with other microphysiological units to examine metabolic and drug interactions (Figure 2). The microphysiological system includes a tissue-engineered blood vessel, 750 to $1,000 \mu \mathrm{m}$ in diameter, in parallel with the muscle tissue. The blood vessel consists of a confluent layer of human endothelial cells and a contractile medial layer of human dermal fibroblasts. The medial layer of the TEBV was fabricated either: from aligned human mesenchymal stem cell sheets rolled into a tubular structure, cultured in a rotating wall bioreactor, followed by maturation in a perfusion bioreactor; or by preparing a tubular structure of dense collagen containing human neonatal dermal fibroblasts that is then cultured in a perfusion chamber for 1 week [22]. TEBV mechanical strength is enhanced using oscillating pressure and flow, similar to approaches used for larger diameter vessels [23-25]. Physiological pulsatile flow is generated using a novel magnetoactive porous ferrogel $[26,27]$ that acts as a valve, changing its hydraulic conductivity under applied magnetic fields to tune the flow rate in the microphysiological system on demand. Other microphysiological organs (for example, myocardium, liver) can be added in parallel to the muscle (Figure 2a). 


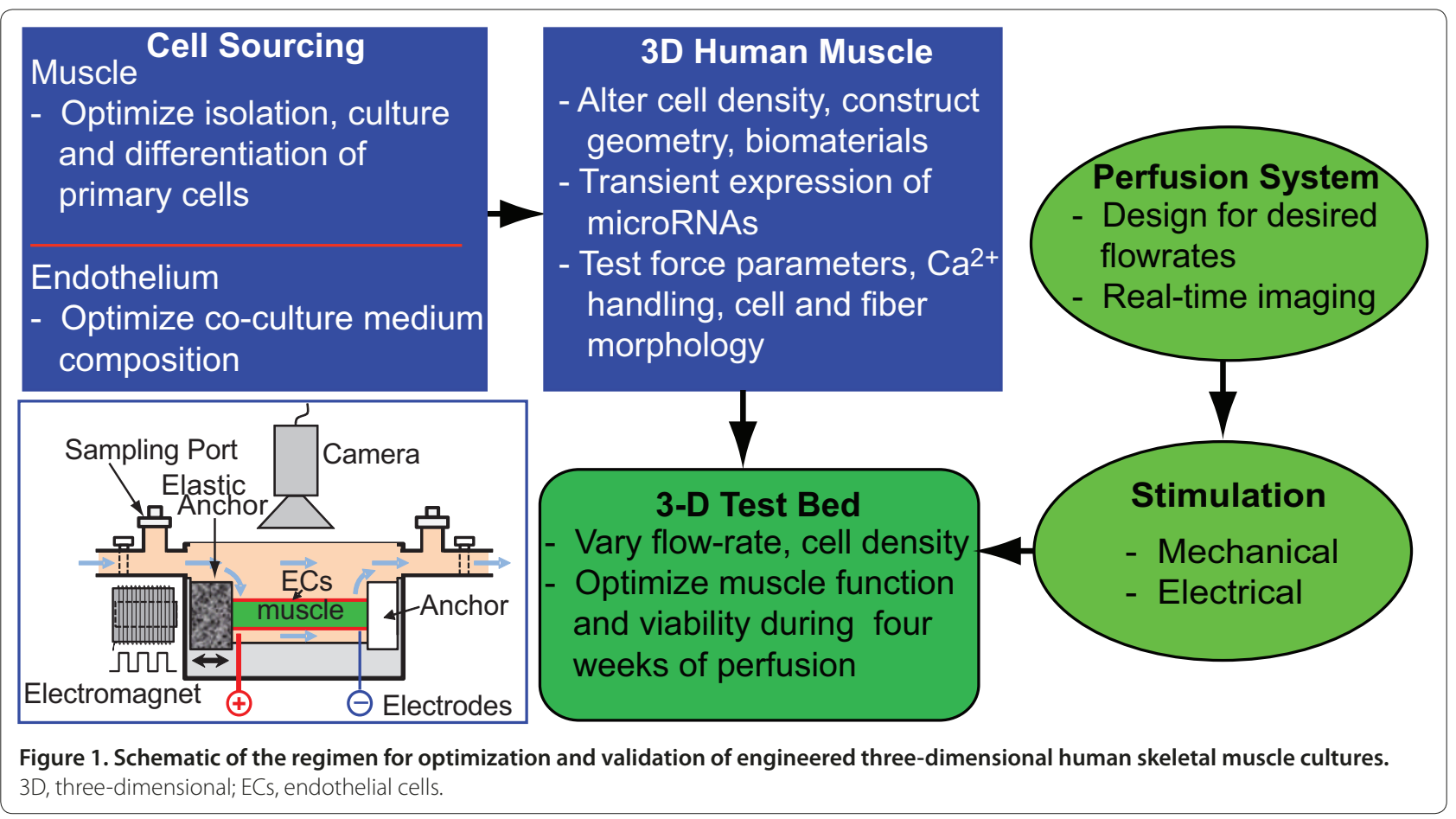

Functional evaluation in the system involves monitoring the mechanical behavior of engineered muscle and blood vessels. The diameter of the engineered blood vessel is measured in response to changes in pressure, from which the incremental elastic modulus and the ultimate tensile strength prior to failure are determined [24]. Vessel dilation in response to changes in flow and nitric oxide release are used to monitor the function of the endothelium. For the muscle tissue, oxygen uptake and the contractile force provide an assessment of muscle function.

The design of the system with human cells requires that the shear stress on endothelium in the blood vessel ranges from 0.4 to $2.0 \mathrm{~Pa}$ [28]; that the rate of oxygen delivery to the muscle equals or exceeds the rate of oxygen uptake by muscle; and that materials used should not bind drugs. Additional requirements for multiorgan systems are that the relative size of different microphysiological organs and their flow rates should be scaled in proportion to in vivo values [1]; that a common media is used for all of the different engineered tissues; and that the system must operate for at least 4 weeks.

Under resting conditions, oxygen uptake in humans in vivo is approximately $1 \times 10^{-8}$ moles $\mathrm{O}_{2} /$ second $/ \mathrm{cm}^{3}$ muscle tissue and can increase 50-fold during exercise [29], similar to our estimate for the oxygen uptake levels of murine skeletal muscle fibers in three-dimensional cultures [13]. Consequently, we assume that the in vivo uptake rates are representative of muscle fibers in vitro. For a muscle cell density of $1 \times 10^{8}$ cells $/ \mathrm{ml}$ and oxygen dissolved in culture medium, the flow rate to muscle tissue under resting conditions needs to be at least $2.54 \mu \mathrm{l} /$ second. Since $21 \%$ of the cardiac output flows through skeletal muscle, the total flow rate in the system is $12 \mu \mathrm{l} /$ second. Adjusting the culture medium viscosity to the blood viscosity, the time-averaged shear stress acting on the endothelium in the TEBV is $0.43 \mathrm{~Pa}$ for a $1,000 \mu \mathrm{m}$ diameter vessel and is $0.84 \mathrm{~Pa}$ for an $800 \mu \mathrm{m}$ diameter vessel, well within the range of values reported in vivo. The flow is laminar, with Reynolds number around 5 for the TEBV, and is quasi-static, similar to conditions in arterioles [30].

Since skeletal muscle is more abundant than other tissues included in an integrated microphysiological system, this flow rate should meet metabolic demands of other tissues - including the liver, which has a high metabolic rate but has 20 times less mass than muscle. Control of flow distribution with magnetoactive valves allows simple interfacing with lower-flow microfluidic platforms and permits adjustment of the flow rate distribution in response to different physiological stimuli. Continuous monitoring of the oxygen concentration in the perfusate combined with a feedback loop can serve to tune flow rate in a physiological-like fashion.

\section{Validation and testing of microphysiological systems}

Validation of the microphysiological system involves measuring vessel relaxation and constriction, skeletal muscle contractile force and metabolism over a 4-week 


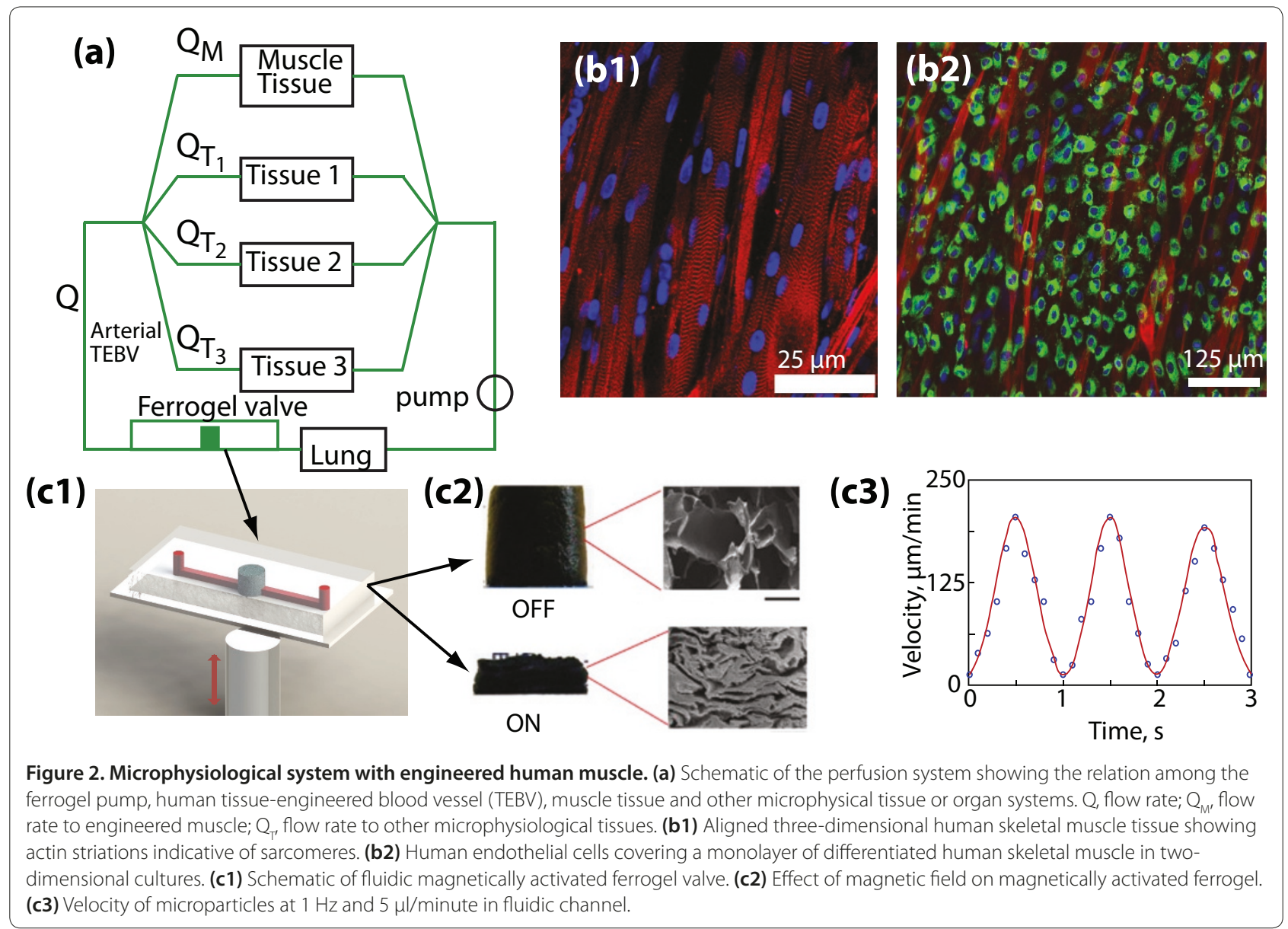

period. The robustness of the system can be assessed by evaluating perturbations from normal physiology, including acute simulated exercise and acute exposure to TNF $\alpha$.

Once the function of the tissues in a microphysiological system is validated, a set of candidate drugs known to affect skeletal muscle and blood vessels, as well as other tissues, can be tested. Compounds to be examined include those that test normal function (phenylephrine), drugs that are safe and effective (lovastatin and metformin [31]), drugs that are effective and unsafe (cerivastatin [32,33]), and compounds with known toxicity to skeletal muscle or blood vessels (for example, antimycin [34], rotenone [34] and doxorubicin [35,36]). Initial results indicate that, based upon contractile force generation, engineered human skeletal muscle bundles are more sensitive to doxorubicin than rodent skeletal muscle fibers [35].

In summary, we have developed the components of a microphysiological system that uses functional measures of blood vessel and skeletal muscle to assess the effect of drugs and toxins. By adjusting cell numbers and flow rates, the system is flexible enough to integrate with other microfluidic and perfusion systems to examine the response of a number of organs and tissues to drugs.

\section{Abbreviations}

miRNA, microRNA; TEBV, tissue-engineered blood vessel; TNF, tumor necrosis factor.

\section{Competing Interests}

The authors declare that they have no competing interests.

\section{Authors' contributions}

GAT designed experiments, discussed interpretation of the results, and wrote the paper. HEA analyzed data, suggested experiments and edited the paper. NB designed and analyzed experiments, wrote portions of the paper, and edited the paper. HFC performed experiments and analyzed data. CSC designed and performed experiments and analyzed data. CF performed experiments and analyzed data. SH designed and performed experiments and analyzed data. YJ designed and performed experiments and analyzed data. TK analyzed results and edited paper. WEK analyzed results and edited paper. $\mathrm{KL}$ designed and analyzed experiments, wrote portions of the paper, and edited the paper. LM designed and analyzed experiments. WMR designed and analyzed experiments, wrote portions of the paper, and edited the paper. XZ designed and analyzed experiments, wrote portions of the paper, and edited the paper. All authors read and approved the final manuscript.

\section{Acknowledgements}

The work was supported by grants R01AR055226 (to NB), R21 AR055195 (to GAT) and UH2TR000505 and the National Institutes of Health Common Fund for the Microphysiological Systems Initiative and Darwin Prockop (NIH grant P40 RR017447) for providing the adult human mesenchymal stem cells. The publication costs of this article were funded by UH2TR000505. 


\section{Declarations}

Publication of this supplement has not been supported by sponsorship. Articles have undergone the journal's standard review process. The Editors declare that they have no competing interests.

This article has been published as part of Stem Cell Research \& Therapy Volume 4 Supplement 1, 2013: Stem cells on bioengineered microphysiological platforms for disease modeling and drug testing. The full contents of the supplement are available online at http://www.stemcellres.com/supplements/4/S1.

\section{Author details}

'Department of Biomedical Engineering, Duke University, 136 Hudson Hall, CB 90281, Durham, NC 27708-0281, USA. ²Department of Surgery, Duke University Medical Center, Durham, NC 27710, USA. ${ }^{3}$ Department of Pathology, Duke University Medical Center, Durham, NC 27710, USA. ${ }^{4}$ Department of Mechanical Engineering and Materials Science, Duke University, Durham, NC 27708-0287, USA. 5Department of Medicine, Duke University Medical Center, Durham, NC 27710, USA.

\section{Published: 20 December 2013}

\section{References}

1. Esch MB, King TL, Shuler ML: The role of body-on-a-chip devices in drug and toxicity studies. Ann Rev Biomed Eng 2011, 13:55-72.

2. Zurlo F, Larson K, Bogardus C, Ravussin E: Skeletal muscle metabolism is a major determinant of resting energy expenditure. J Clin Invest 1990 , 86:1423-1427.

3. Evans WJ, Paolisso G, Abbatecola AM, Corsonello A, Bustacchini S, Strollo F, Lattanzio F: Frailty and muscle metabolism dysregulation in the elderly. Biogerontology 2010, 11:527-536.

4. Iannuzzi-Sucich M, Prestwood KM, Kenny AM: Prevalence of sarcopenia and predictors of skeletal muscle mass in healthy, older men and women. J Gerontol A Bio/ SciMed Sci 2002, 57:M772-M777.

5. Khamseh ME, Malek M, Aghili R, Emami Z: Sarcopenia and diabetes: pathogenesis and consequences Br J Diabetes Vasc Dis 2011, 11:230-234.

6. Brass EP, Sietsema KE: Considerations in the development of drugs to treat sarcopenia. J Am Geriatr Soc 2011, 59:530-535.

7. Evans WJ: Drug discovery and development for ageing: opportunities and challenges. Philos Trans R Soc B Biol Sci 2011, 366:113-119.

8. Dykens JA, Will Y: The significance of mitochondrial toxicity testing in drug development. Drug Discov Today 2007, 12:777-785.

9. Torok M, Zahno A, Waldhauser KM, Brecht K, S. Kraahenbuhl S: Toxicity of statins on rat skeletal muscle mitochondria. Cell Mol Life Sci 2006, 63:2415-2425.

10. Brown MA, Wallace CS, Angelos M, Truskey GA: Characterization of umbilical cord blood-derived late-outgrowth endothelial progenitor cells exposed to laminar shear stress. Tissue Eng Part A 2009, 15:3575-3587.

11. Guo X, Gonzalez M, Stancescua M, Vandenburgh HH, Hickman JJ: Neuromuscular junction formation between human stem cell-derived motoneurons and human skeletal muscle in a defined system. Biomaterials 2011, 32:9602-9611.

12. Zhang SJ, Truskey GA, Kraus WE: Effect of cyclic stretch on $\beta 1 D$-integrin expression and activation of FAK and RhoA. Am J Physio/ Cell Physio/ 2007 292:C2057-C2069.

13. Rhim C, Lowell DA, Reedy MC, Slentz DH, Zhang SJ, Kraus WE, Truskey GA Morphology and ultrastructure of differentiating three-dimensional mammalian skeletal muscle in a collagen gel. Muscle Nerve 2007, 36:71-80.

14. Liao IC, Liu JB, Bursac N, Leong KW: Effect of electromechanical stimulation on the maturation of myotubes on aligned electrospun fibers. Cell Mol Bioeng 2008, 1:133-145.

15. Bian W, Bursac N: Engineered skeletal muscle tissue networks with controllable architecture. Biomaterials 2009, 30:1401-1412.

16. Bian W, Bursac N: Soluble miniagrin enhances contractile function of engineered skeletal muscle. Faseb J 2011, 26:955-965.

17. Hinds S, Bian W, Dennis RG, Bursac N: The role of extracellular matrix composition in structure and function of bioengineered skeletal muscle. Biomaterials 2011, 32:3575-3583
18. Bian W, Juhas M, Pfeiler TW, Bursac N: Local tissue geometry determines contractile force generation of engineered muscle networks. Tissue Eng Part A 2012, 18:957-967.

19. Trappe S, Williamson D, Godard M, Porter D, Rowden G, Costill D: Effect of resistance training on single muscle fiber contractile function in older men. J Appl Physiol 2000, 89:143-152.

20. Rhim C, Cheng CS, Kraus WE, Truskey GA: Effect of microRNA modulation on bioartificial muscle function. Tissue Eng Part A 2010, 16:3589-3597.

21. Aoi W, Naito Y, Mizushima K, Takanami Y, Kawai Y, Ichikawa H, Yoshikawa T: The microRNA miR-696 regulates PGC-1a in mouse skeletal muscle in response to physical activity. Am J Physiol Endocrinol Metab 2010, 298:E799-E806.

22. Ghezzi CE, Risse PA, Marelli B, Muja N, Barralet JE, Martin JG, Nazhat SN: An airway smooth muscle cell niche under physiological pulsatile flow culture using a tubular dense collagen construct. Biomaterials 2013, 34:1954-1966.

23. Kumar VA, Brewster LP, Caves JM, Chaikof EL: Tissue engineering of blood vessels: functional requirements, progress, and future challenges. Cardiovasc Eng Technol 2011, 2:137-148.

24. Syedain ZH, Meier LA, Bjork JW, Lee A, Tranquillo RT: Implantable arterial grafts from human fibroblasts and fibrin using a multi-graft pulsed flow-stretch bioreactor with noninvasive strength monitoring. Biomaterials 2011, 32:714-722.

25. Zaucha MT, Raykin J, Wan W, Gauvin R, Auger FA, Germain L, Michaels TE, Gleason RL: A novel cylindrical biaxial computer-controlled bioreactor and biomechanical testing device for vascular tissue engineering. Tissue Eng Part A 2009, 15:3331-3340.

26. Zang J, Ryu J, Pugno N, Wang Q, Tu Q, Buehler MJ, Zhao X: Multifunctionality and control of the crumpling and unfolding of large-area graphene. Nat Mater 2012, 12:321-325.

27. Zhao XH, Kim J, Cezar CA, Huebsch N, Lee K, Bouhadir K, Mooney DJ: Active scaffolds for on-demand drug and cell delivery. Proc Natl Acad Sci U S A 2011, 108:67-72.

28. Chiu JJ, Chien S: Effects of disturbed flow on vascular endothelium: pathophysiological basis and clinical perspectives. Physiol Rev 2011 91:327-387.

29. Blomstrand E, Radegran G, Saltin B: Maximum rate of oxygen uptake by human skeletal muscle in relation to maximal activities of enzymes in the Krebs cycle. J Physiol 1997, 501:455-460.

30. Truskey GA, Yuan F, Katz DF: Transport Phenomenon in Biological Systems. 2nd edition. Upper Saddle River, NJ: Pearson/Prentice Hall; 2009.

31. Sarabia V, Lam L, Burdett E, Leiter LA, Klip A: Glucose transport in human skeletal muscle cells in culture. Stimulation by insulin and metformin. $J$ Clin Invest 1992, 90:1386-1395.

32. Vandenburgh H, Shansky J, Benesch-Lee F, Barbata V, Reid J, Thorrez L, Valentin $\mathrm{R}$, Crawford G: Drug-screening platform based on the contractility of tissueengineered muscle. Muscle Nerve 2008, 37:438-447.

33. Kaufmann P, Török M, Zahno A, Waldhauser KM, Brecht K, Krahenbuhl S: Toxicity of statins on rat skeletal muscle mitochondria. Cell Mol Life Sci 2006, 63:2415-2425.

34. Shaham O, Slatea NG, Goldbergera O, Xud O, Ramanathanb A, Souzab AL, Clishb CB, Simsa KB, Moothaa VK: A plasma signature of human mitochondrial disease revealed through metabolic profiling of spent media from cultured muscle cells. Proc Natl Acad Sci U S A 2010, 107:1571-1575.

35. van Norren K, van Helvoort A, Argiles JM, van Tuijl S, Arts K, Gorselink M, Laviano A, Kegler D, Haagsman HP, van der Beek EM: Direct effects of doxorubicin on skeletal muscle contribute to fatigue. Br J Cancer 2009, 100:311-314

36. Gilliam LA, St Clair DK: Chemotherapy-induced weakness and fatigue in skeletal muscle: the role of oxidative stress. Antioxid Redox Signal 2011 $15 \cdot 2543-2563$

doi:10.1186/scrt37

Cite this article as: Truskey GA, et al: Design considerations for an integrated microphysiological muscle tissue for drug and tissue toxicity testing. Stem Cell Research \& Therapy 2013, 4(Suppl 1):S10 